\title{
TOPOLOGICAL INVARIANTS OF WEIGHTED HOMOGENEOUS POLYNOMIALS
}

\author{
by ZBIGNIEW SZAFRANIEC
}

(Received 23 January, 1990)

Let $f: \mathbb{R}^{n} \rightarrow \mathbb{R}$ be a weighted homogeneous polynomial such that $d f(0)=0$, let $L=\left\{x \in S^{n-1} \mid f(x)=0\right\}$, and let $\chi(L)$ be the Euler characteristic of $L$. The problem is how to calculate $\chi(L)$ in terms of $f$.

In the paper we shall show that there are maps $H_{1}, H_{2}:\left(\mathbb{R}^{n}, 0\right) \rightarrow\left(\mathbb{R}^{n}, 0\right)$ defined explicitly in terms of $f$ such that $0 \in \mathbb{R}^{n}$ is isolated in $H_{i}^{-1}(0), i=1,2$, and

$$
\chi(L)=2-\left(\operatorname{deg}_{0}\left(H_{1}\right)+\operatorname{deg}_{0}\left(H_{2}\right)+\chi\left(S^{n-1}\right)\right),
$$

where $\operatorname{deg}_{0}\left(H_{i}\right)$ is the topological degree of the map

$$
x \mapsto H_{i}(x) /\left\|H_{i}(x)\right\|
$$

from a small sphere centered at the origin to $S^{n-1}$. If $f$ is a homogeneous polynomial then the above formula is a consequence of results which have been proved in [6], [7], [8].

The paper has been written at the Department of Mathematics of the University of New Mexico in Albuquerque. I am very grateful to Professor Wojciech Kucharz for the opportunity to work here.

We start with a technical lemma.

Lemma 1. Let $\omega_{1}, \omega_{2}, f:\left(\mathbb{R}^{n}, 0\right) \rightarrow(\mathbb{R}, 0)$ be polynomials such that $\omega_{1}>0, \omega_{2}>0$ everywhere, except at the origin, and $\lim \omega_{1}(x)=\lim \omega_{2}(x)=+\infty$ for $\|x\| \rightarrow+\infty$. Let $N_{r}^{i}=\left\{x \mid \omega_{i}(x)=r, f(x) \leq 0\right\}$. If $r>0$ is small enough then $H_{*}\left(N_{r}^{1}, \mathbb{Z}_{2}\right) \cong H_{*}\left(N_{r}^{2}, \mathbb{Z}_{2}\right)$. In particular $\chi\left(N_{r}^{1}\right)=\chi\left(N_{r}^{2}\right)$.

Proof. Let $M_{r}^{i}=\left\{x \mid 0<\omega_{i}(x) \leq r, f(x) \leq 0\right\}, i=1,2$. According to the local triviality of polynomial mappings (see [3]) there is $r_{0}>0$ such that $\omega_{i}: M_{r_{0}}^{i} \rightarrow\left(0, r_{0}\right]$ is a trivial fibration. Since $\omega_{i} \geq 0, \omega_{i}^{-1}(0)=\{0\}$, and $\omega_{i}(x) \rightarrow+\infty$ as $\|x\| \rightarrow+\infty$, there are constants $r_{1}, r_{2}$ such that $0<r_{2}<r_{1}<r_{0}$ and $M_{r_{2}}^{1} \subset M_{r_{1}}^{2} \subset M_{r_{0}}^{1}$. From the triviality of $\omega_{1} \mid M_{r_{0}}^{1}$, the induced homomorphism $H_{*}\left(M_{r_{2}}^{1}, \mathbb{Z}_{2}\right) \rightarrow H_{*}\left(M_{r_{0}}^{1}, \mathbb{Z}_{2}\right)$ is an isomorphism. Hence the induced homomorphism $H_{*}\left(M_{r_{2}}^{1}, \mathbb{Z}_{2}\right) \rightarrow H_{*}\left(M_{r_{1}}^{2}, \mathbb{Z}_{2}\right)$ is injective. From the triviality of $\omega_{i} \mid M_{r_{0}}^{i}$ we may deduce that $H_{*}\left(M_{r_{2}}^{1}, \mathbb{Z}_{2}\right) \cong H_{*}\left(M_{r_{0}}^{1}, \mathbb{Z}_{2}\right), H_{*}\left(M_{r_{1}}^{2}, \mathbb{Z}_{2}\right) \cong H_{*}\left(M_{r_{0}}^{2}, \mathbb{Z}_{2}\right)$ and so there is an injection $H_{*}\left(M_{r_{0}}^{1}, \mathbb{Z}_{2}\right) \rightarrow H_{*}\left(M_{r_{0}}^{2}, \mathbb{Z}_{2}\right)$. Using similar arguments we may prove that there exists the opposite injection, and then $H_{*}\left(M_{r_{0}}^{1}, \mathbb{Z}_{2}\right) \cong H_{*}\left(M_{r_{0}}^{2}, \mathbb{Z}_{2}\right)$. From the triviality of $\omega_{i} \mid M_{r_{0}}^{i}$ we also know that $N_{r_{0}}^{i}$ is a deformation retract of $M_{r_{0}}^{i}$, and then $H_{*}\left(N_{r_{0}}^{1}, \mathbb{Z}_{2}\right) \cong H_{*}\left(N_{r_{0}}^{2}, \mathbb{Z}_{2}\right)$.

Let $d_{1}, \ldots, d_{n} \geq 1$ be positive integers. For every $z=\left(z_{1}, \ldots, z_{n}\right) \in \mathbb{C}^{n}$ and $\lambda \in \mathbb{C}$ we shall denote $\lambda . z=\left(\lambda^{d_{1}} z_{1}, \ldots, \lambda^{d_{n}} z_{n}\right)$.

Let $f: \mathbb{R}^{n} \rightarrow \mathbb{R}$ be a weighted homogeneous polynomial such that $d f(0)=0$ and $f(\lambda . x)=\lambda^{d} f(x), d \geq 2$, for every $x \in \mathbb{R}^{n}, \lambda \in \mathbb{R}$. Let $p$ be the smallest positive integer such that $2 p>d$ and each $d_{i}$ divides $p$. 
Let $a_{i}=p / d_{i}$ and let

$$
\omega=\omega\left(x_{1}, \ldots, x_{n}\right)=\frac{x_{1}^{2 a_{1}}}{2 a_{1}}+\ldots+\frac{x_{n}^{2 a_{n}}}{2 a_{n}} .
$$

Clearly $\omega>0$ except at the origin. Let $g_{1}=f-\omega$ and let $I_{1} \subset \mathbb{R}\left[\left[x_{1}, \ldots, x_{n}\right]\right]$ be the ideal generated by $\partial g_{1} / \partial x_{1}, \ldots, \partial g_{1} / \partial x_{n}$. Since $d f(0)=0, I_{1}$ is a proper ideal.

Lemma 2. $\operatorname{dim} \mathbb{R}\left[\left[x_{1}, \ldots, x_{n}\right]\right] / I_{1}<\infty$.

Proof. Because $f$ is a weighted homogeneous polynomial,

$$
\lambda^{d_{i}} \frac{\partial f}{\partial x_{i}}(\lambda . z)=\lambda^{d} \frac{\partial f}{\partial x_{i}}(z)
$$

thus

$$
\frac{\partial f}{\partial x_{i}}(\lambda . z)=\lambda^{d-d_{i}} \frac{\partial f}{\partial x_{i}}(z)
$$

for every $z=\left(z_{1}, \ldots, z_{n}\right) \in \mathbb{C}^{n}, \lambda \in \mathbb{C}$. Hence

$$
\frac{\partial g_{1}}{\partial x_{i}}(\lambda . z)=\lambda^{d-d_{i}}\left(\frac{\partial f}{\partial x_{i}}(z)-\lambda^{2 a_{i} d_{i}-d} z_{i}^{2 a_{i}-1}\right)
$$

According to (1) we have

$$
2 a_{i} d_{i}-d=2 p-d \geq 1
$$

Now it is easy to see that the set

$$
\bigcap_{i=1}^{n}\left\{(z, \lambda) \in \mathbb{C}^{n} \times \mathbb{C} \mid\|z\|=1, \frac{\partial g_{1}}{\partial x_{i}}(\lambda . z)=0\right\}
$$

is compact in $\mathbb{C}^{n} \times \mathbb{C}$. The continuous map $S^{2 n-1} \times \mathbb{C} \ni(z, \lambda) \mapsto \lambda . z \in \mathbb{C}^{n}$ is onto, so $\left\{z \in \mathbb{C}^{n} \mid \operatorname{grad} g_{1}(z)=0\right\}$ is an algebraic, compact, and so finite subset of $\mathbb{C}^{n}$. This means that $0 \in \mathbb{C}^{n}$ is an isolated point in the set of critical points of $g_{1}$, and so

$$
\operatorname{dim}_{\mathbb{R}} \mathbb{R}\left[\left[x_{1}, \ldots, x_{n}\right]\right] / I_{1}=\operatorname{dim}_{\mathbb{C}} \mathbb{C}\left[\left[z_{1}, \ldots, z_{n}\right]\right] /\left(\frac{\partial g_{1}}{\partial x_{1}}, \ldots, \frac{\partial g_{1}}{\partial x_{n}}\right)<\infty .
$$

Let $H_{1}=\operatorname{grad} g_{1}:\left(\mathbb{R}^{n}, 0\right) \rightarrow\left(\mathbb{R}^{n}, 0\right)$. According to Lemma $2,0 \in \mathbb{R}^{n}$ is isolated in $H_{1}^{-1}(0)$, and so $\operatorname{deg}_{0}\left(H_{1}\right)$ is well-defined.

Let $A_{1}=\left\{x \in S^{n-1} \mid f(x) \leq 0\right\}$. Then we have

LEMMA 3. $\chi\left(A_{1}\right)=1-\operatorname{deg}_{0}\left(H_{1}\right)$.

If $f$ is a homogeneous polynomial of degree $d$ then $d_{1}=\ldots=d_{n}=1, p=[d / 2]+1$ and $\omega=\left(x_{1}^{2 p}+\ldots+x_{n}^{2 p}\right) / 2 p$. In that case the above formula has been proved in [7].

Proof. Set

$$
V=\left\{(x, r, y) \in \mathbb{R}^{n} \times \mathbb{R} \times \mathbb{R} \mid \omega(x)=r^{2 p}, \operatorname{rank}(d \omega(x), d f(x)) \leq 1, y=f(x)\right\} .
$$

Thus $V$ is an algebraic subset of $\mathbb{R}^{n} \times \mathbb{R} \times \mathbb{R}$. Put $\Sigma_{r}=\left\{x \in \mathbb{R}^{n} \mid \omega(x)=r^{2 p}\right\}$, where $r \neq 0$. Clearly $\Sigma_{r}$ is a smooth manifold diffeomorphic to $S^{n-1}$. Let $\pi: \mathbb{R}^{n} \times \mathbb{R} \times \mathbb{R} \rightarrow \mathbb{R} \times \mathbb{R}$ be 
the natural projection. If $r \neq 0$ then

$$
\pi(V) \cap\{r\} \times \mathbb{R}=\{r\} \times\left\{\text { critical values of } f \mid \Sigma_{r}\right\} .
$$

The set of critical values of a polynomial mapping restricted to an algebraic manifold is finite (see [5, p.16]), so $\pi(V) \cap\{r\} \times \mathbb{R}$ is finite.

Let us take $(x, r, y) \in V$ and $\lambda \in \mathbb{R}$. Then

$$
\begin{array}{r}
\omega(\lambda . x)=\frac{\left(\lambda^{d_{1}} x_{1}\right)^{2 a_{1}}}{2 a_{1}}+\ldots+\frac{\left(\lambda^{d_{n}} x_{n}\right)^{2 a_{n}}}{2 a_{n}} \\
=\frac{\lambda^{2 d_{1} a_{1}} x_{1}^{2 a_{1}}}{2 a_{1}}+\ldots+\frac{\lambda^{2 d_{n} a_{n}} x_{n}^{2 a_{n}}}{2 a_{n}} \\
=\lambda^{2 p} \omega(x)=(\lambda r)^{2 p}, \\
\operatorname{det}\left[\begin{array}{ll}
\frac{\partial \omega}{\partial x_{i}}(\lambda . x) & \left.\frac{\partial \omega}{\partial x_{j}}(\lambda . x)\right] \\
\frac{\partial f}{\partial x_{i}}(\lambda . x) & \frac{\partial f}{\partial x_{j}}(\lambda . x)
\end{array}\right] \\
=\operatorname{det}\left[\begin{array}{cc}
\lambda^{d_{i}\left(2 a_{i}-1\right)} \frac{\partial \omega}{\partial x_{i}}(x) & \lambda^{d_{j}\left(2 a_{j}-1\right)} \frac{\partial \omega}{\partial x_{j}}(x) \\
\lambda^{d-d_{i}} \frac{\partial f}{\partial x_{i}}(x) & \lambda^{d-d_{j}} \frac{\partial f}{\partial x_{j}}(x)
\end{array}\right] \\
=\operatorname{det}\left[\begin{array}{cc}
\lambda^{2 p-d_{i}} \frac{\partial \omega}{\partial x_{i}}(x) & \lambda^{2 p-d_{j}} \frac{\partial \omega}{\partial x_{j}}(x) \\
\lambda^{d-d_{i}} \frac{\partial f}{\partial x_{i}}(x) & \lambda^{d-d_{j}} \frac{\partial f}{\partial x_{j}}(x)
\end{array}\right] \\
=\lambda^{2 p+d-d_{i}-d_{i}} \operatorname{det}\left[\begin{array}{ll}
\frac{\partial \omega}{\partial x_{i}}(x) & \frac{\partial \omega}{\partial x_{j}}(x) \\
\frac{\partial f}{\partial x_{i}}(x) & \frac{\partial f}{\partial x_{j}}(x)
\end{array}\right]=0
\end{array}
$$

because $\operatorname{rank}(d \omega(x), d f(x)) \leq 1$. Hence $\operatorname{rank}(d \omega(\lambda . x), d f(\lambda . x)) \leq 1$ too. We have

$$
f(\lambda . x)=\lambda^{d} f(x)=\lambda^{d} y .
$$

So, if $(x, r, y) \in V$ then $\left(\lambda . x, \lambda r, \lambda^{d} y\right) \in V$ too. Hence $\pi(V)$ is a finite union of curves and if $(r, y) \in \pi(V)$ and $\lambda \in \mathbb{R}$ then $\left(\lambda r, \lambda^{d} y\right) \in \pi(V)$. Because $2 p>d$,

$$
|y|>r^{2 p}
$$

for every point $(r, y) \in \pi(V), y \neq 0$, sufficiently close to the origin. Set

$$
V^{\prime}=\left\{(x, r, y) \mid \omega(x)=r^{2 p}, \operatorname{rank}\left(d \omega(x), d g_{1}(x)\right) \leq 1, y=g_{1}(x)\right\} .
$$

Since $g_{1}=f-\omega$, we have $\operatorname{rank}\left(d \omega(x), d g_{1}(x)\right)=\operatorname{rank}(d \omega(x), d f(x))$, and then

$$
V^{\prime}=\left\{(x, r, y) \mid \omega(x)=r^{2 p}, \operatorname{rank}(d \omega(x), d f(x)) \leq 1, y=f(x)-r^{2 p}\right\} .
$$


Define $\Theta(r, y)=\left(r, y-r^{2 p}\right)$. Then

$$
\pi\left(V^{\prime}\right)=\Theta(\pi(V))
$$

Let $N=\left\{x \in \Sigma_{r} \mid f(x) \leq 0\right\}, N_{1}=\left\{x \in \Sigma_{r} \mid g_{1}(x) \leq 0\right\}=\left\{x \in \Sigma_{r} \mid f(x) \leq \omega(x)\right\}$. Clearly $N \subset \operatorname{int}\left(N_{1}\right)$.

From (iv) and (v), if $r>0$ is small enough then the function $g_{1} \mid \Sigma_{r}$ has no critical points in $N_{1}-N$. In particular, the function $g_{1}$ has an isolated critical point at the origin. The set $N$ is closed, semialgebraic and hence, according to [4], can be triangulated. So $N$ is a deformation retract of $N_{1}$, and then $\chi(N)=\chi\left(N_{1}\right)$. Let $M_{1}=\left\{x \mid\|x\|=r, g_{1}(x) \leq 0\right\}$. According to [1], [9], $\chi\left(M_{1}\right)=1-\operatorname{deg}_{0}\left(H_{1}\right)$. Of course $M_{1}=\left\{x \mid\|x\|^{2 p}=r^{2 p}, g_{1}(x) \leq 0\right\}$, $N_{1}=\left\{x \mid \omega(x)=r^{2 p}, g_{1}(x) \leq 0\right\}$ and then from Lemma 1 we have $\chi\left(M_{1}\right)=\chi\left(N_{1}\right)$ (where $\left.\omega_{1}=\|x\|^{2 p}, \omega_{2}=\omega\right)$. The polynomial $f$ is weighted homogeneous and so $\chi\left(A_{1}\right)=\chi(N)$. Hence $\chi\left(A_{1}\right)=1-\operatorname{deg}_{0}\left(H_{1}\right)$.

Let $g_{2}=-(f+\omega)=-f-\omega, \quad$ let $\quad A_{2}=\left\{x \in S^{n-1} \mid f(x) \geq 0\right\}, \quad$ and let $I_{2} \subset$ $\mathbb{R}\left\{\left[x_{1}, \ldots, x_{n}\right]\right\}$ be the ideal generated by $\partial g_{2} / \partial x_{1}, \ldots, \partial g_{2} / \partial x_{n}$. Using the same arguments as above we can prove that $\operatorname{dim} \mathbb{R}[[x]] / I_{2}<\infty$, so $0 \in \mathbb{R}^{n}$ is isolated in $H_{2}^{-1}(0)$, where $H_{2}=\operatorname{grad} g_{2}:\left(\mathbb{R}^{n}, 0\right) \rightarrow\left(\mathbb{R}^{n}, 0\right)$. Clearly $A_{2}=\left\{x \in S^{n-1} \mid-f(x) \leq 0\right\}$, so from Lemma 3 we have

LEMMA 4. $\chi\left(A_{2}\right)=1-\operatorname{deg}_{0}\left(H_{2}\right)$.

Let $L=\left\{x \in S^{n-1} \mid f(x)=0\right\}$. Since $L=A_{1} \cap A_{2}$ and $S^{n-1}=A_{1} \cup A_{2}$, we have $\chi(L)=$ $\chi\left(A_{1}\right)+\chi\left(A_{2}\right)-\chi\left(S^{n-1}\right)$; thus

THEOREM 5. $\chi(L)=2-\left(\operatorname{deg}_{0}\left(H_{1}\right)+\operatorname{deg}_{0}\left(H_{2}\right)+\chi\left(S^{n-1}\right)\right)$.

If $d$ is odd then $\tau: \tau(x)=(-1) \cdot x$ is an involution on $S^{n-1}$ such that $f(\tau(x))=$ $(-1)^{d} f(x)=-f(x)$. It follows that $\tau\left(A_{1}\right)=A_{2}$ and then $A_{1}$ is homeomorphic to $A_{2}$. From Lemmas 3, 4 and Theorem 5 we have

COROllary 6. If $d$ is odd then

$$
\chi(L)=2\left(1-\operatorname{deg}_{0}\left(H_{1}\right)\right)-\chi\left(S^{n-1}\right) .
$$

According to Lemma 2, dimensions of local algebras associated to $H_{1}, H_{2}$ are finite, so we may compute $\operatorname{deg}_{0}\left(H_{i}\right)$ using the Eisenbud-Levine algorithm (see [2]). In the following examples we shall apply a computer program by Andrzej Łecki from the Institute of Mathematics in Gdańsk which is able to calculate $\operatorname{deg}_{0}\left(H_{i}\right)$ using that algorithm.

The author wants to express his gratitude to Andrzej Łęcki for his cooperation.

EXAMPLE 1. Let $f(x, y, z)=x^{2} y-y^{4}-y z^{3}$. The polynomial $f$ is weighted homogeneous, where $d_{1}=3, d_{2}=d_{3}=2, d=8$. Clearly $p=6$ has the property (1), and so $a_{1}=2$, $a_{2}=a_{3}=3$. Hence $\omega=x^{4} / 4+y^{6} / 6+z^{6} / 6$, and

$$
\begin{aligned}
& H_{1}=\operatorname{grad}(f-\omega)=\left(2 x y-x^{3}, x^{2}-4 y^{3}-z^{3}-y^{5},-3 y z^{2}-z^{5}\right), \\
& H_{2}=\operatorname{grad}(-f-\omega)=\left(-2 x y-x^{3},-x^{2}+4 y^{3}+z^{3}-y^{5}, 3 y z^{2}-z^{5}\right) .
\end{aligned}
$$

Thanks to the computer program by Andrzej Łęcki we have been able to calculate that $\operatorname{deg}_{0}\left(H_{1}\right)=\operatorname{deg}_{0}\left(H_{2}\right)=1$. From Theorem 5, $\chi(L)=-2$. Since

$$
L=\left\{(x, y, z) \in S^{2} \mid y\left(x^{2}-y^{3}-z^{3}\right)=0\right\}
$$


is homeomorphic to a union of two circles with two common points, the solution is correct.

EXAmple 2. Let $f(x, y, z)=x^{3}+x^{2} z-y^{2}$. The polynomial $f$ is weighted homogeneous, where $d_{1}=d_{3}=2, d_{2}=3, d=6$. Then $p=6, a_{1}=a_{3}=3, a_{2}=2$ and $\omega=x^{6} / 6+$ $y^{4} / 4+z^{6} / 6$. Hence

$$
\begin{aligned}
& H_{1}=\operatorname{grad}(f-\omega)=\left(3 x^{2}+2 x z-x^{5},-2 y-y^{3}, x^{2}-z^{5}\right), \\
& H_{2}=\operatorname{grad}(-f-\omega)=\left(-3 x^{2}-2 x z-x^{5}, 2 y-y^{3},-x^{2}-z^{5}\right) .
\end{aligned}
$$

A computer has calculated that $\operatorname{deg}_{0}\left(H_{1}\right)=1, \operatorname{deg}_{0}\left(H_{2}\right)=-1$, and so $\chi(L)=0$. The reader may easily check that the solution is correct.

EXAMPLE 3. Let $f(x, y, z)=x^{3}-x y^{2}+x y z+2 x^{2} y-2 y^{3}-y^{2} z-x z^{2}+y z^{2}$. The polynomial $f$ is homogeneous of degree $d=3$, so $d_{1}=d_{2}=d_{3}=1, p=2, a_{1}=a_{2}=a_{3}=2$ and $\omega=\left(x^{4}+y^{4}+z^{4}\right) / 4$. Hence $H_{1}=\left(3 x^{2}-y^{2}+y z+4 x y-z^{2}-x^{3},-2 x y+x z+2 x^{2}-6 y^{2}-\right.$ $\left.2 y z+z^{2}-y^{3}, x y-y^{2}-2 x z+2 y z-z^{3}\right)$. A computer has calculated that $\operatorname{deg}_{0}\left(H_{1}\right)=3$. According to Corollary $6, \chi(L)=-6$. The reader may check that $f=(x-y)(x+y+z)$ $(x+2 y-z)$, so the solution is correct.

\title{
REFERENCES
}

1. V. I. Arnold, Index of a singular point of a vector field, the Petrovski-Oleinik inequality, and mixed hodge structures, Functional Anal. Appl. 2 (1978), 1-11.

2. D. Eisenbud and H. I. Levine, An algebraic formula for the degree of a $C^{\infty}$ map germ, Ann. of Math. (2) 7 (1977), 19-44.

3. R. Hardt, Semi-algebraic local triviality in semi-algebraic mappings, Amer. J. Math. 102 (1980), 291-302.

4. S. Lojasiewicz, Triangulation of semi-analytic sets, Ann. Scuola Norm. Sup. Pisa (3) 18 (1964), 449-474.

5. J. Milnor, Singular points of complex hypersurfaces (Princeton University Press, 1968).

6. Z. Szafraniec, On the Euler characteristic of analytic and algebraic sets, Topology 25 (1986), 411-414.

7. Z. Szafraniec, On the Euler characteristic of complex algebraic varieties, Math. Ann. 280 (1988), 177-183.

8. Z. Szafraniec, On the Euler characteristic mod 2 of real projective varieties, Math. Proc. Cambridge Philos. Soc. 104 (1988), 479-481. 345-350.

9. C. T. C. Wall, Topological invariance of the Milnor number mod 2, Topology 22 (1983),

\author{
INSTITUTE OF MATHEMATICS \\ UNIVERSITY OF GDAŃSK \\ 80-952 GDAŃSK \\ Wita Stwosza 57 \\ Poland
}

